\title{
CONTROLLING OF DEPTH OF DOPANT DIFFUSION LAYER IN A MATERIAL BY TIME MODULATION OF DIFFUSION COEFFICIENT
}

\author{
E.L. Pankratov ${ }^{1}$ and E.A. Bulaeva ${ }^{1,2}$ \\ ${ }^{1}$ Nizhny Novgorod State University, 23 Gagarin avenue, Nizhny Novgorod, 603950, \\ Russia \\ ${ }^{2}$ Nizhny Novgorod State University of Architecture and Civil Engineering, 65 Il'insky \\ street, Nizhny Novgorod, 603950, Russia
}

\begin{abstract}
In this paper as a development of recently introduced analytical approach for estimation of temporal characteristics of mass and heat transport we present analysis of diffusion depth of dopant in a material with time varying diffusion coefficient. It has been shown, that changing of time dependence of diffusion coefficient gives a possibility to accelerate or decelerate diffusion process. In this situation it is an actual question is control of diffusion depth during manufacturing $p$ - $n$-junctions. The controlling gives a possibility to obtain required depth of the junctions, but not larger or smaller.
\end{abstract}

\section{KEYWORDS}

Time varying diffusion coefficient; diffusion depth of dopant; control of diffusion depth

\section{INTRODUCTION}

One of main technological processes to manufacture of semiconductor devices is dopant diffusion at high temperature [1]. Increasing of temperature of annealing required to increase dopant diffusion coefficient. In this situation dopant diffusion coefficient increases to manufacture $p-n$ junctions and other solid state electronic device to accelerate dopant diffusion. At final stage of technological process temperature of annealing should be decreased to decrease dopant diffusion coefficient and to finish technological process. Framework the technological process a dopant should be infused in a homogeneous sample or in an epitaxial layer on required depth at varying in time of temperature of annealing. Varying in time temperature of annealing leads to varying in time of diffusion coefficient. However influence of law of varying in time of diffusion coefficient on depth of dopant has been weakly investigated. Most often analysis of dopant diffusion has been done at constant diffusion coefficient [1,2]. However replacing of time-varying diffusion coefficient by constant one is sometime not adequate [3-5]. Main aim of the present paper is analysis of influence of nonstationary diffusion coefficient on diffusion depth of dopant. The accompanying aim of the present paper is determination of conditions on variation in time diffusion coefficient, which correspond to acceleration and deceleration of dopant diffusion. 


\section{Method of Solution}

To analyze influence of nonstationary diffusion coefficient on diffusion depth and determination of required conditions we determine spatio-temporal distribution of concentration of dopant $C$ $(x, t)$ by solving the following the second Fick's law $[1,2]$

$$
\frac{\partial C(x, t)}{\partial t}=D(t) \frac{\partial^{2} C(x, t)}{\partial x^{2}} .
$$

Boundary and initial conditions could be written as

$$
C(0, t)=C_{0},\left.\frac{\partial C(x, t)}{\partial x}\right|_{x=L}=0, C(x, 0)=0 .
$$

Here $C(x, t)$ is the spatio-temporal distribution of concentration of dopant; $D(t)$ is the timedependence dopant diffusion coefficient. Boundary condition on the left side gives a possibility to take into account infusion of dopant through the boundary. Boundary condition on the left side gives a possibility to take into account absents of dopant flow through the boundary. Initial condition describes absents of dopant in the considered material before starting of doping. First of all we consider small variation of dopant diffusion coefficient in time. Analysis of dopant diffusion in this case gives a possibility to obtain more demonstrative results. In the considered case dopant diffusion depends first of all by average value of diffusion coefficient $D_{0}$. To simplify analysis of dopant diffusion we transform diffusion coefficient $D(t)$ to the following form: $D$ $(t)=D_{0}[1+\mu \cdot v(t)], 0 \leq \mu<<1,|v(t)| \leq 1$ is the modulation depth of the diffusion coefficient [4,5]. After that we determine solution of diffusion coefficient as the following power series

$$
C(x, t)=\sum_{k=0}^{\infty} \mu^{k} C_{k}(x, t) .
$$

Substitution of the series into Eq. (1) and groping of coefficients at equal powers of parameter $\mu$ in left and right sides gives a possibility to obtain equations for functions $C_{k}(x, t)$

$$
\left\{\begin{array}{l}
\frac{\partial C_{0}(x, t)}{\partial t}=D_{0} \frac{\partial^{2} C_{0}(x, t)}{\partial x^{2}} \\
\frac{\partial C_{k}(x, t)}{\partial t}=D_{0} \frac{\partial^{2}\left[C_{k}(x, t)+v(t) C_{k-1}(x, t)\right]}{\partial x^{2}}, k \geq 1 .
\end{array}\right.
$$

Initial and boundary conditions for the functions $C_{k}(x, t)$ could be written with account decomposition (3) as

$$
C_{0}(0, t)=\alpha_{0}, C_{k \geq 1}(0, t)=0,\left.\frac{\partial C_{k \geq 0}(x, t)}{\partial x}\right|_{x=L}=0, C_{k \geq 0}(x>0,0)=0 .
$$

Zero-order approximation of concentration of dopant, which correspond to material with averaged diffusion coefficient, is the solution of the first equation of system (4) by using the Fourier approach 


$$
C_{0}(x, t)=\alpha_{0}\left[1-\frac{2}{\pi} \sum_{n=0}^{\infty} \frac{1}{n+0.5} \sin \left(a_{n} x\right) \exp \left(-a_{n}^{2} D_{0} t\right)\right]
$$

where $a=(n+0.5) \pi / L$. Solution of the diffusion equation with constant diffusion equation could be generalized to the case with time-varying diffusion coefficient by using the following replacement of the product $D_{0} t$ on the integral $\int_{0}^{t} D(u) d u$ [6]. The replacement transforms the relation (5) to the following form

$$
C(x, t)=\alpha_{0}\left[1-\frac{2}{\pi} \sum_{n=0}^{\infty} \frac{1}{n+0.5} \sin \left(a_{n} x\right) \exp \left(-a_{n}^{2} \int_{0}^{t} D(u) d u\right)\right] .
$$

However using functions $C_{k}(x, t)$ give a possibility to make more demonstrative analysis of diffusion process in comparison with using the relation (6). To analyze influence of nonstationary diffusion coefficient on diffusion depth of dopant it is attracted an interest to use the first-order correction to dopant concentration, because the correction usually gives a possibility to make most demonstrative analysis. The correction could be obtained by solving the second equation of the system (4) by using the Fourier approach or by decomposition of the relation (6) in the power series of parameter $\mu$

$$
C_{1}(x, t)=2 \alpha_{0} \frac{D_{0}}{L} \int_{0}^{t} v(u) d u \sum_{n=0}^{\infty} a_{n} \sin \left(a_{n} x\right) \exp \left(-a_{n}^{2} D_{0} t\right) .
$$

Now we estimate diffusion depth of dopant in material. We determine the diffusion depth $\ell(t)$ framework the approach from [7], i.e. as the interval between boundary of material $x=0$ (we consider the boundary as source of dopant) and coordinate of step-wise changing of function, which has been used for approximation of concentration of dopant $C(x, t)$ with minimal mean-squared error. We used the following approximation function: $\psi(x, t, \ell(t))=a_{0}(t)+a_{1}(t)[1(t)-1(t-\ell(t))]$. Optimal values of parameters $a_{0}(t), a_{1}(t)$ and $\ell(t)$ have been obtain by minimization of the meansquared error

$$
U=\int_{0}^{L}[C(x, t)-\psi(x, t, \ell)]^{2} d x
$$

A necessary condition for the extreme of the mean-squared error $U$ on the parameters $a_{0}, a_{1}$ and $\ell$ is

$$
\frac{\partial U}{\partial a_{0}}=0, \frac{\partial U}{\partial a_{1}}=0, \frac{\partial U}{\partial \ell}=0
$$

The first condition leads to the following equation to determine the considered parameters

$$
\int_{0}^{L} C(x, t) d x=a_{0}(t) L+a_{1}(t) \ell(t)
$$


Minimization of the error $U$ by $a_{1}$ и $\ell$ leads to the following equations to determine the considered parameters

$$
\begin{gathered}
\int_{0}^{L} C(x, t) d x=\left[a_{0}(t)+a_{1}(t)\right] \ell(t), \\
C(\ell(t), t)=a_{0}(t)+a_{1}(t) / 2 .
\end{gathered}
$$

The considered criterion of estimation of diffusion depth is nonlinear. The nonlinearity is not a problem for analysis of physical results. Increasing of dimension of the considered sample $L$ leads to correction of parameters $a_{0}, a_{1}$ and $\ell$.

To obtain analytical results asymptotically optimal criterion is attracted an interest. Framework the asymptotically optimal criterion the condition should be taken into account $L>>\ell(t)$ [7]. At the same time one can obtain for the case: $a_{1}(t)=\alpha_{0}-C(x, 0)$ and $a_{2}(t)=\alpha_{0}$. In this situation it should be determined only coordinate of step-wise changing of approximation function $\psi$. In this situation we obtain linear relation for estimation of the coordinate $\ell(t)$ as the rectangle with equal square $[4,5,7]$

$$
\ell(t)=\frac{1}{\alpha_{0}} \int_{0}^{L} C(x, t) d x
$$

Linearity of the criterion (13) on concentration and decomposition (3) gives a possibility to use superposition principle and present diffusion depth of dopant into sample as a power series on parameter $\mu$

$$
\ell(t)=\ell_{0}(t)\left[1+\sum_{n=1}^{\infty} \mu^{n} \chi_{1}(t)\right]
$$

where $\ell_{0}(t)=\frac{1}{\alpha_{0}} \int_{0}^{L} C_{0}(x, t) d x$ is the zero-order approximation of diffusion depth, functions $\chi_{i}(t)=\int_{0}^{L} C_{i}(x, t) d x / \int_{0}^{L} C_{0}(x, t) d x$ are the normalized corrections of the diffusion depth. Substitution of solutions of diffusion equation (5) and (7) into the series (14) leads to the following results

$$
\begin{aligned}
& \ell_{0}(t)=L\left[1-\frac{2}{\pi^{2}} \sum_{n=0}^{\infty} \frac{\exp \left(-a_{n}^{2} D_{0} t\right)}{(n+0.5)^{2}}\right], \\
& \chi_{1}(t)=\frac{2 D_{0} \int_{0}^{t} v(u) d u \sum_{n=0}^{\infty} \exp \left(-a_{n}^{2} D_{0} t\right)}{L^{2}\left[1-\frac{2}{\pi^{2}} \sum_{n=0}^{\infty} \frac{\exp \left(-a_{n}^{2} D_{0} t\right)}{(n+0.5)^{2}}\right]} .
\end{aligned}
$$




\section{Example of Calculation of Diffusion Depth}

Diffusion coefficient depend on temperature framework Arrhenius law [9,10]: $D(T)=D \cdot \exp [-$ $E / k T$, where $D$ is the pre-exponential multiplayer, $E$ is the activation energy, $k$ is the Boltzmann constant, $T$ is the temperature of material. Time dependence of temperature of material during heating and cooling could be approximated by the following function: $T(t)=T_{k}+T_{0}[1-\exp (-t / \tau)]$ $\left[1(t)-1\left(t-t_{0}\right)\right]+T_{0}\left[1-\exp \left(-t_{0} / \tau\right)\right] \exp (-t / \tau)\left[1(t)-1\left(t-t_{0}-3 \tau\right)\right]$, where $1(t)$ is the unit function; $T_{k}$ is the initial temperature (in our case is the room temperature); $\tau$ is the time scale of heating and cooling of material, which depends on speed of heat transport; $t_{\mathrm{o}}$ is the continuance of heating of material; temperature of heating $T_{0}$ depends on power $P$ of source of heat. The temperature could be estimated as $T_{0}=P t_{\mathrm{d}} / C$, where $C$ is the heat capacitance of material. We assume, that at the time scale $t \sim t_{\mathrm{o}}$ achieves its maximal value $T_{k}+T_{0}$, i.e. $t_{0} \geq 3 \tau$. In this situation dependence of dopant diffusion coefficient on time at high-temperature doping could be written as

$$
D(t) \approx D \exp \left\{-E / k\left[T_{k}+T_{0}[1-\exp (-t / \tau)]\left[1(t)-1\left(t-t_{0}\right)\right]+T_{0} \exp \left(-\left(t-t_{0}\right) / \tau\right)\left[1\left(t-t_{0}\right)-1\left(t-t_{0}-3 \tau\right)\right]\right]\right\}
$$

where $D_{0}=\frac{D^{\theta}}{\theta} \int_{0}^{\exp }\left\{-E / k\left[T_{k}+T_{0}\left[1-\exp \left(-\frac{t}{\tau}\right)\right]\left[1(t)-1\left(t-t_{o}\right)\right]+T_{0} \exp \left(-\frac{t-t_{0}}{\tau}\right)\left[1\left(t-t_{o}\right)-1(t-\theta)\right]\right\} d t, \theta\right.$ $=t_{0}+3 \tau$, value of the parameter $\mu$ depends on value of temperature $T_{0}$ : increasing of the temperature leads to increasing of the parameter $\mu$. Linearity of the correction $\chi_{1}(t)$ on function $v(t)$ gives a possibility to use spectral decomposition [11] of the function $v(t)$ on orthogonal system of functions. Usually harmonic functions are used for spectral for the spectral decomposition. We consider decomposition of time dependence of dopant diffusion coefficient into discrete Fourier series with period $\theta=t_{0}+3 \tau$. Linearity of dopant diffusion depth on function $v(t)$ for spectral components $v(t)=\cos (\omega t+\varphi)$ with account relation (17) gives the possibility to obtain for the firstorder correction for diffusion depth $\chi_{1}(t)$ the following relation

$$
\chi_{1}(t)=\frac{2 D_{0}[\sin (\omega t+\varphi)-\sin \varphi] \sum_{n=0}^{\infty} \exp \left(-a_{n}^{2} D_{0} t\right)}{L^{2} \omega\left[1-\frac{2}{\pi^{2}} \sum_{n=0}^{\infty} \frac{\exp \left(-a_{n}^{2} D_{0} t\right)}{(n+0.5)^{2}}\right]} .
$$

Fig. 1 shows dependences of the correction (18) on frequency of spectral components $\omega$ for different values of initial phase $\varphi$. For zero value of the initial phase one can obtain increasing of value of dopant diffusion coefficient. In this situation one can obtain increasing of value of correction $\chi_{1}(t)$ to diffusion depth (see curve 1 ). If the initial phase is equal to $\pi / 2$ one can not obtain any changing of values of dopant diffusion coefficient and correction $\chi_{1}(t)$ (see curve 2). If the initial phase is equal to $\pi$ or to $3 \pi / 2$ one can obtain changing of sign of spectral component $v$ $(t)=\cos (\omega t+\varphi)$ with appropriate changing of dopant diffusion coefficient and correction $\chi_{1}(t)$ (see curves 3 and 4 ).

\section{Conclusions}

In this paper we introduce a criterion to estimate diffusion depth of dopant in a material with varying in time diffusion coefficient. Based on this criterion we analyzed diffusion depth of dopant in the material as a functional of varying in time diffusion coefficient. We obtain conditions to 
acceleration and deceleration of dopant diffusion in the material. In this situation we obtain conditions to increase or to decrease (depends on choosing of time varying law) the above depth.

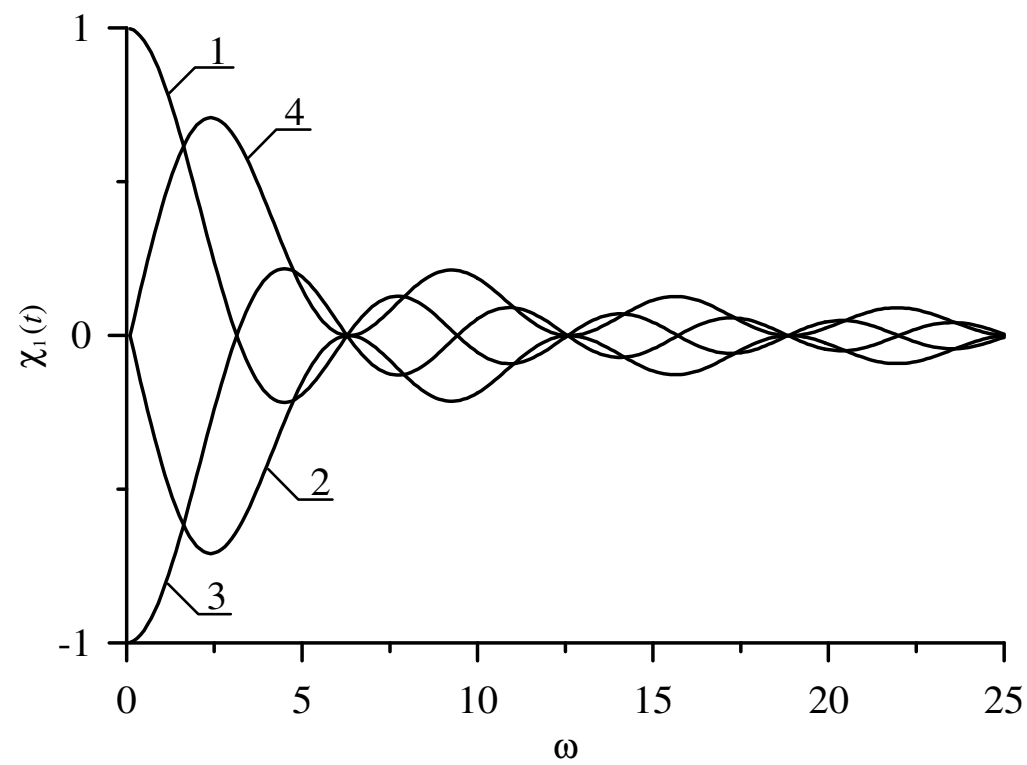

Fig. 1. Dependences of correction to the diffusion depth $\chi_{1}(t)$ on frequency of spectral components of dopant diffusion coefficient

\section{ACKNOWLEDGEMENTS}

This work is supported by the agreement of August 27, 2013 № 02.B.49.21.0003 between The Ministry of education and science of the Russian Federation and Lobachevsky State University of Nizhni Novgorod, educational fellowship for scientific research of Government of Russian, educational fellowship for scientific research of Government of Nizhny Novgorod region of Russia and educational fellowship for scientific research of Nizhny Novgorod State University of Architecture and Civil Engineering.

\section{REFERENCES}

[1] B.A. Zon, S.B. Ledovsky, A.N. Likholet. Lattice diffusion of impurity enhanced by a surface heterogeneous reaction. Technical Physics. Vol. 70 (4). P. 419-422 (2000).

[2] O.V. Alexandrov, A.A. Krivoruchko, N.A. Sobolev. Simulation of aluminum diffusion in silicon in inert and oxidizing media. Semiconductors. Vol. 40 (4). P. 379-384 (2006).

[3] A.V. Yanilkin. Quantum molecular dynamics simulation of hydrogen diffusion in zirconium hydride. Physics of the Solid State. Vol. 56 (9). P. 1879-1885 (2014).

[4] E.L. Pankratov. Controlling the diffusion process via time-variable diffusion coefficient. Technical Physics. Vol. 74 (1). P. 114-118 (2004).

[5] E.L. Pankratov, E.A. Bulaeva. Optimal criteria to estimate temporal characteristics of diffusion process in a media with inhomogenous and nonstationary parameters. analysis of influence of variation of diffusion coefficient on values of time characteristics. Rev. Theor. Sci. Vol. 1 (3). P. 58-82 (2013).

[6] A.I. Raichenko. Mathematical theory of diffusion in applications (Kiev: Naukova Dumka, 1981).

[7] A.N. Malakhov, E.L. Pankratov. Relaxation time of material density in a medium with arbitrary space-varying diffusion coefficient and potential profile. Radiophysics and Quantum Electronics. Vol. 44 (4). P. 357-363 (2001). 
International Journal on Cybernetics \& Informatics (IJCI) Vol. 5, No. 3, June 2016

[8] I.P. Stepanenko. Basis of microelectronics (Moscow, Soviet Radio, 1980).

[9] A.E. Dolbak, R.A. Zhachuk, B.A. Ol'shanetsky. Mechanism of copper diffusion over the Si(110) surface. Semiconductors. Vol. 36 (9). P. 958-961 (2002).

[10] W.P. Gillin, D.J. Dunstan. Strain and interdiffusion in semiconductor heterostructures. Phys. Rev.B. Vol. 50 (11). P. 7495-7498 (1994).

[11] G. Korn, T. Korn. Mathematical Handbook for scientists and engineers. Definitions, theorems and formulas for reference and review (Second edition. McGraw-Hill Book Company. New York, 1968).

[12] A.N. Malakhov, A.L. Pankratov. Evolution times of probability distributions and averaged-exact solutions of the Kramer's problem. Adv. Chem. Phys. Vol. 121. P. 356-438 (2002).

\section{AUTHORS}

Pankratov Evgeny Leonidovich was born at 1977. From 1985 to 1995 he was educated in a secondary school in Nizhny Novgorod. From 1995 to 2004 he was educated in Nizhny Novgorod State University: from 1995 to 1999 it was bachelor course in Radiophysics, from 1999 to 2001 it was master course in Radiophysics with specialization in Statistical Radiophysics, from 2001 to 2004 it was PhD course in Radiophysics. From 2004 to 2008 E.L. Pankratov was a leading technologist in Institute for Physics of Microstructures. From 2008 to 2012 E.L. Pankratov was a senior lecture/Associate Professor of Nizhny Novgorod State University of Architecture and Civil Engineering. 2012-2015 Full Doctor course in Radiophysical Department of Nizhny Novgorod State University. Since 2015 E.L. Pankratov is an Associate Professor of Nizhny Novgorod State University. He has 160 published papers in area of his researches.

Bulaeva Elena Alexeevna was born at 1991. From 1997 to 2007 she was educated in secondary school of village Kochunovo of Nizhny Novgorod region. From 2007 to 2009 she was educated in boarding school "Center for gifted children". From 2009 she is a student of Nizhny Novgorod State University of Architecture and Civil Engineering (spatiality "Assessment and management of real estate"). At the same time she is a student of courses "Translator in the field of professional communication" and "Design (interior art)" in the University. Since 2014 E.A. Bulaeva is in a PhD program in Radiophysical Department of Nizhny Novgorod State University. She has 107 published papers in area of her researches. 